Research paper

\title{
New and improved methods for measuring lymphocyte proliferation in vitro and in vivo using CFSE-like fluorescent dyes
}

\author{
Benjamin J.C. Quah *, Christopher R. Parish \\ Department of Immunology, John Curtin School of Medical Research, Australian National University, Canberra, ACT 2601, Australia
}

\section{A R T I C L E I N F O}

\section{Article history:}

Received 25 January 2012

Accepted 13 February 2012

Available online 21 February 2012

\section{Keywords:}

Carboxyfluorescein diacetate

succinimidyl ester

Flow cytometry

Fluorescent dyes

Lymphocyte division

\begin{abstract}
A B S T R A C T
The use of carboxyfluorescein diacetate succinimidyl ester (CFSE) to measure lymphocyte proliferation by flow cytometry has become one of the most widely utilised assays for assessing lymphocyte responses. The properties of CFSE make it ideal for such a task, covalently labelling cells with a long-lived fluorescence of high intensity and low variance with minimal cell toxicity. No dye in the last 20 years has been capable of replicating CFSE in these respects. However, currently CFSE is limited to following a maximum of 7 cell divisions and is not compatible for use with ubiquitously available fluorescein conjugates or other fluorescent molecules with spectral properties similar to fluorescein, such as EGFP. Here we characterise two new fluorescent dyes for measuring lymphocyte proliferation, Cell Trace Violet (CTV) and Cell Proliferation Dye eFluor 670 (CPD), which have different excitation and emission spectra to CFSE and, consequently, are compatible with fluorescein conjugates. We found that while both CTV and CPD can label cells to a high fluorescence intensity, which is long-lived and has low variability and low toxicity and makes them ideal for long-term tracking of nondividing lymphocytes in vivo, CTV offers possibly the best available alternative to CFSE in the analysis of cell divisions. We also describe how intercellular dye transfer and cell autofluorescence can affect division resolution with the three different dyes and describe labelling conditions for the three dyes that produce ultra-bright lymphocytes for in vivo tracking studies and allow up to 11 cell divisions to be detected when using CFSE and CTV as the fluorescent dyes.
\end{abstract}

(C) 2012 Elsevier B.V. All rights reserved.

\section{Introduction}

The development of an adaptive immune response relies on a rapid expansion in the number of lymphocytes specific for a foreign antigen. Therefore, measurement of lymphocyte expansion using sensitive but convenient methods is critical for the progress of immunological research. The measurement of lymphocyte proliferation, both in vitro and in vivo, took a dramatic leap forward following the discovery of the cell

Abbreviations: CFSE, carboxyfluorescein diacetate succinimidyl ester; CTV, Cell Trace Violet;CPD, Cell Proliferation Dye eFluor 670

* Corresponding author at: Department of Immunology, John Curtin School of Medical Research, Building 131, Garran Road, The Australian National University, Canberra ACT 2601, Australia. Tel.: +61 26125 4716; fax: + 61261252595 .

E-mail address: ben.quah@anu.edu.au (B.J.C. Quah). labelling properties of the dye carboxyfluorescein diacetate succinimidyl ester (CFSE) which was ideal for flow cytometry assays (Weston and Parish, 1990; Lyons and Parish, 1994). Since then CFSE has been used in numerous studies on lymphocyte function (Hodgkin et al., 1996; Kurts et al., 1997; Bird et al., 1998; Gett and Hodgkin, 1998; Nordon et al., 1999; Marzo et al., 2000; Jedema et al., 2004; Stambas et al., 2007). In fact, since its initial description as a lymphocyte tracking and proliferation dye, CFSE has been used in over 20,000 scientific publications and has been cited in almost 1000 patents.

The use of CFSE in cell division analysis relies on the simple premise that when a dye-labelled cell divides, the CFSE fluorescence intensity is halved in the two daughter cells. However, in order to achieve high resolution of each cell division, it is essential that the labelling dye meets several important characteristics, namely (i) high initial staining intensity 
which allows numerous cell divisions to be detected before the dye dilutes to levels approaching cellular autofluorescence, (ii) low fluorescence variance, in order to maintain discrete division peaks, (iii) minimal and uniform dye leakage over long periods, so that fluorescence intensity and variance are not compromised, and (iv) low cell toxicity to ensure that cell function is not altered by the labelling procedure. Although numerous dyes have met some of these labelling characteristics, none have been able to match the unique properties of CFSE (Parish, 1999).

The remarkable success of CFSE is based on two main chemical attributes of the dye (Parish, 1999; Quah and Parish, 2010). The first is the presence of two acetate groups, which allows the dye to rapidly cross the plasma membrane of cells. However, removal of the acetate groups by intracellular esterases lessens the membrane permeability of the dye, thus allowing the dye to concentrate within cells. The second important chemical attribute of CFSE is the amino-reactive succinimidyl side chain of the dye, which allows CFSE to covalently couple to numerous intracellular proteins, including those with a low turnover rate. This results in a high degree of stable fluorescence, with CFSE typically allowing up to 8 peaks of fluorescence (or 7 cell divisions) to be measured by flow cytometry before the dye dilutes to cellular autofluorescence levels (Quah et al., 2007). When labelling is performed quickly, the rapid reactivity of CFSE also results in a population of lymphocytes with a low variance of fluorescence, typically with a standard deviation of each division peak being $\sim 20 \%$ or less of the mean fluorescence intensity (Quah et al., 2007). This allows clear visualisation of discrete fluorescence peaks following each cell division. Although CFSE labelling under some conditions can affect cell behaviour (Quah et al., 2007; Last'ovicka et al., 2009; Parish et al., 2009), when used to label cells in appropriate buffering conditions it has very low cellular toxicity (Quah et al., 2007; Parish et al., 2009).

One of the main disadvantages of CFSE is that it removes the use of one of the most commonly used fluorescence channels in flow cytometry, i.e., the channel used for fluorescein detection. An additional disadvantage of CFSE is that, based on current labelling procedures, a maximum of $\sim 7$ cell divisions can be measured. An added incentive to develop proliferation dyes with different spectral properties to CFSE is that it would allow the proliferation of different populations of lymphocytes to be simultaneously measured in the same culture or animal. Recently, two new fluorescent dyes have become available that seem to have the potential to work as well as CFSE but possess different fluorescence excitation and emission spectra. Cell Trace Violet (referred to here as CTV), produced by Molecular Probes, is excited by violet emissions $(405 \mathrm{~nm})$ commonly used in violet laser-equipped flow cytometers. It emits at a peak wavelength of $\sim 450 \mathrm{~nm}$ and thus can be used in flow cytometers equipped with detectors for Pacific Blue. It is claimed to be capable of detecting up to 8-10 cell divisions, although this is based on results from flow cytometers equipped with acoustic focusing, this procedure being capable of generating better fluorescence peak resolution than standard flow cytometers (Molecular Probes, Invitrogen; Applied Biosystems Life Technologies). The second dye, Cell Proliferation Dye eFluor 670 (referred to here as CPD), produced by eBioscience, has a peak excitation of $647 \mathrm{~nm}$ and can be detected using the APC fluorochrome detectors. It is claimed by the manufacturer to be capable of detecting up to 5 cell divisions. Although these dyes appear to be promising candidates for measuring lymphocyte proliferation, there are no available reports comparing them side by side with CFSE.

In this report we have examined the ability of CTV and CPD to detect the proliferation of mouse lymphocyte in vitro and in vivo in comparison with CFSE, focusing on the key virtues of CFSE, namely, fluorescence intensity, low fluorescence variance, high stability of cell labelling and low cellular toxicity. It was found that CTV is comparable to CFSE in monitoring lymphocyte proliferation but CPD was a less effective dye, although both new dyes stably label lymphocytes and are potentially as useful as CFSE for long-term tracking of non-dividing lymphocytes in vivo. During this comparative study, we also identified intercellular dye transfer and cell autofluorescence as two potential causes of reduced resolution of cell division peaks using CFSE and CFSE-like dyes. In addition, we describe an optimised methodology for cell labelling with all three fluorescent dyes that results in ultrabright lymphocytes for cell migration studies and increases the number of cell division detectable, potentially up to 11 , when employing commonly used flow cytometers.

\section{Methods}

\subsection{Animals}

Mice were obtained from the Australian National University (ANU) Bioscience Services, ANU. Mice were housed and handled according to the guidelines of the ANU Animal Experimentation Ethics Committee. Mouse strains used were C57BL/6 (B6), B6.CD45.1 (B6 congenic for CD45.1) and the transgenic ( $\mathrm{Tg}$ ) mouse strains MD4 (B cell receptor (BCR)-Tg expressing hen egg lysozyme (HEL)-specific-IgM ${ }^{\mathrm{a}}$ and IgD $^{\mathrm{a}}$ on a B6 background (Goodnow et al., 1988)), OT-II (T cell receptor (TCR)Tg specific for I-A $\mathrm{A}^{\mathrm{b}}$-ovalbumin (OVA) $)_{323-339}$ peptide on a B6 background (Barnden et al., 1998)), P14.CD45.1 (TCR-Tg specific for $\mathrm{D}^{\mathrm{b}}-\mathrm{GP}_{33-41}$ peptide (lymphocytic choriomeningitis virus-derived) on a B6.CD45.1 background (Pircher et al., 1989)), $\mathrm{F} 5$ (TCR-Tg specific for $\mathrm{D}^{\mathrm{b}}$ - $\mathrm{NP}_{366-374}$ peptide (influenza virus-derived) on a B6 background (Mamalaki et al., 1993)) and OT-I (TCR-Tg specific for $\mathrm{K}^{\mathrm{b}}-\mathrm{OVA}_{257-264}$ peptide on a $\mathrm{B} 6$ background (Hogquist et al., 1994)). Male mice were used at $6-12$ weeks of age.

\subsection{Lymphocyte preparation}

Lymphocytes were obtained from spleen and/or lymph nodes as previously described (Quah et al., 2004). CD4 ${ }^{+} \mathrm{T}$ cells and $\mathrm{CD}^{+} \mathrm{T}$ cells were enriched from pooled lymph nodes as well as spleen, and B cells were enriched from spleen via magnetic-activated cell sorting (MACS) (Miltenyi Biotec) as previously described (Quah et al., 2004).

\subsection{Labelling lymphocytes with fluorescent dyes}

CFSE, CTV (both from Molecular Probes, Invitrogen) and CPD (eBioscience) fluorescent dyes were dissolved in DMSO as $10 \mathrm{mM}$ stock solutions (stored at $-20^{\circ} \mathrm{C}$ ). For dye labelling lymphocytes were resuspended to $1 \times 10^{8} / \mathrm{mL}$ in $20^{\circ} \mathrm{C}$ RPMI 1640 medium (Invitrogen) supplemented with $10 \%$ 
foetal calf serum (FCS) and a final concentration of $10-80 \mu \mathrm{M}$ of each dye added to $1 \mathrm{~mL}$ aliquots of lymphocytes with immediate vortexing to ensure rapid and homogeneous labelling of cells. Cells were incubated at $20^{\circ} \mathrm{C}$ for $5 \mathrm{~min}$, then washed 3 times with RPMI 1640 supplemented with $10 \%$ FCS. This labelling procedure is as described previously (Quah et al., 2007), using the method videoed by JoVE (Quah and Parish, 2010), with one important change being that the labelling solution used was RPMI 1640 supplemented with 10\% FCS rather than PBS, the use of culture medium and the inclusion of high FCS ensuring that cell toxicity was minimised, particularly when very high concentrations of the fluorescent dyes were being used.

\subsection{In vitro lymphocyte proliferation assays}

Fluorescent dye-labelled lymphocytes were cultured at a concentration of $2-4 \times 10^{6} / \mathrm{mL}$ in RPMI 1640 supplemented with $10 \%$ FCS, $10 \mathrm{mM}$ HEPES, $1 \mathrm{mM}$ glutamine, $1 \mathrm{mM}$ Napyruvate and $55 \mu \mathrm{M} 2-\mathrm{ME}$ in a total volume of $200 \mu \mathrm{L}$ in 96 well U-bottomed plates (Nunc). Polyclonal stimulation involved the use of a cocktail consisting of concanavalin A (ConA, Sigma; $2.5 \mu \mathrm{g} / \mathrm{mL}$ ), lipopolysaccharide (LPS, Sigma; $2.5 \mu \mathrm{g} / \mathrm{mL}$ ), CpG ODN 1668 (GeneWorks; $0.25 \mu \mathrm{g} / \mathrm{mL}$ ), interleukin (IL)-2 (PeproTech; $0.25 \mathrm{ng} / \mathrm{mL}$ ) and IL-4 (PeproTech; $0.25 \mathrm{ng} / \mathrm{mL}$ ). This is referred to as "100\% stimuli" and was often varied by diluting the stimulation cocktail and creating a stimuli titrations for in vitro cultures. Cells were cultured at $37^{\circ} \mathrm{C}$ in $5 \% \mathrm{CO}_{2}$ for $1-4$ days before antibody staining and analysis by flow cytometry.

\subsection{In vivo lymphocyte proliferation assays}

Fluorescent dye-labelled lymphocytes were adoptively transferred into the lateral tail vein of host B6.CD45.1 or B6 mice at a maximum cell number of $5 \times 10^{7}$ in $200 \mu \mathrm{L}$ of PBS using a 29 gauge needle and syringe. Approximately 10-60 min post adoptive transfer, host mice were challenged with antigen in the form of a 1-10 $\mu$ g bolus of a HEL-OVA conjugate (Quah et al., 2008), a $25 \mu \mathrm{g}$ bolus of OVA (Sigma), a $10 \mu \mathrm{mol}$ bolus of the $\mathrm{GP}_{33-41}$ peptide and/or a $50 \mu \mathrm{mol}$ bolus of the $\mathrm{NP}_{366-374}$ peptide in $100 \mu \mathrm{L}$ of PBS into the lateral tail vein. In some experiments, a 1-10 $\mu$ g bolus of LPS was coinjected with antigen to act as a danger signal and improve lymphocyte responses. After 3-6 days cells from spleens of host mice were harvested and prepared for staining and analysis by flow cytometry.

\subsection{Flow cytometry procedures and data analysis}

Antibodies used to delineate lymphocyte populations by flow cytometry included anti-CD4-Alexa Fluor 700 (or biotinylated), anti-CD8 $\alpha$-APC-eFluor 780, anti-B220-PerCP-Cy 5.5 (or Alexa Fluor 700 conjugated), anti-CD45.1-PE-Cy7, anti-CD45.2-PerCP-Cy5.5, anti-IgM ${ }^{\mathrm{a}}$-PE (for MD4 B cells detection), anti-V $\alpha_{2}-\mathrm{PE}$ (for OT-I, OT-II and P14 T cell detection), and anti-Vß $\beta_{11}$-bio (for $\mathrm{F} 5 \mathrm{~T}$ cell detection) and were purchased from either BD Bioscience, eBioscience or Biolegend. Cell viability was assessed with the dye Hoechst $33258(1 \mu \mathrm{g} / \mathrm{ml}$, Calbiochem-Behring Corp.). Cells were stained with antibodies and Hoechst 33258 for 20-30 min on ice then washed twice, as previously described (Quah et al., 2004). Where necessary, the second step reagent SAVPE-Alexa Fluor 610 (Invitrogen) was used to detect biotinylated antibodies, which was incubated with cells for 20-30 min on ice and then cells washed twice. With some in vivo samples, prior to the antibody-staining step, cells were reacted for 25 min at $37^{\circ} \mathrm{C}$ with MHC-I tetramers to aid CD8 ${ }^{+} \mathrm{T}$ cell delineation and then the cells washed twice before antibody staining. MHC-I tetramers used were C9M (KAVYNFATM)peptide- $\mathrm{D}^{\mathrm{b}}$-APC (for P14 TCR detection), $\mathrm{NP}_{366-374}$ (ASNENMDAM)-peptide- $\mathrm{D}^{\mathrm{b}}$-APC (for F5 TCR detection), and $\mathrm{OVA}_{257-264}$ (SIINFEKL)-peptide-K ${ }^{\mathrm{b}}$-APC (for OT-I TCR detection). In order to estimate total cell number in in vitro assays, 5000 FlowCount Fluorospheres (Beckman Coulter) were added, with the antibody staining solution, to cells in the wells of 96 well plates. Relative recovery of beads was then used to estimate relative recovery of cells in each well at the end of the assay.

Analytical flow cytometry was performed with a Fortessa or LSR-II flow cytometer (Becton Dickinson, Mountain View, CA). A log scale was used for the SSC-A profile to allow discrimination of Flow-Count Fluorospheres from lymphocytes in in vitro samples. Post acquisition gating was used to analyse cell subsets as previously described (Quah et al., 2008) using flowJo software (Tree Star, OR). Statistical analysis of the proportion of a cell population expressing dye was based on histogram profiling using Overton subtraction (Overton, 1988) as applied by Flowjo software. The geometric mean (geomean) and co-efficient of variance (CV) was determined by FlowJo software. It was found that the CV of some data sets was dramatically skewed by a small number of events (typically as few as 3 events, or $<0.5 \%$ of the data set). In such cases, these events were classified as outliers and were removed from the data set for $\mathrm{CV}$ generation.

\section{Results}

3.1. CFSE, CTV and CPD label lymphocytes with a similar fluorescence intensity of low variability and high longevity

In order to compare the capacity of CFSE, CTV and CPD to label lymphocytes, mouse splenocytes were labelled with $10 \mu \mathrm{M}$ of each dye in RPMI 1640 culture medium containing $10 \%$ FCS to reduce dye toxicity. Using this labelling method cells were assessed for fluorescence emission over 3 days of in vitro culture by flow cytometry (Fig. 1). Intriguingly, when gated on viable lymphocyte singlets, both CTV-labelled and CPD-labelled splenocyte preparations contained two distinct subpopulations of highly fluorescent cells whereas CFSE labelled splenocytes contained only one distinct population of highly fluorescent cells (Fig. 1a). Similar to CFSE, the CTV and CPD labelled cells displayed a fluorescence intensity $\sim 2.5-3$ logs above background autofluorescence, which gradually declined over the 3 days of in vitro culture, with CPD fluorescence decreasing slightly more rapidly than CFSE and CFSE fluorescence declining slightly more rapidly than CTV (Supplementary Fig. 1). To determine if the two distinct fluorescent peaks seen with CTV and CPD labelled splenocytes were based on lymphocyte subset differences, the labelled lymphocyte populations were further delineated into $\mathrm{CD} 4^{+} \mathrm{T}$ cells, $\mathrm{CD} 8^{+} \mathrm{T}$ cells, 
a
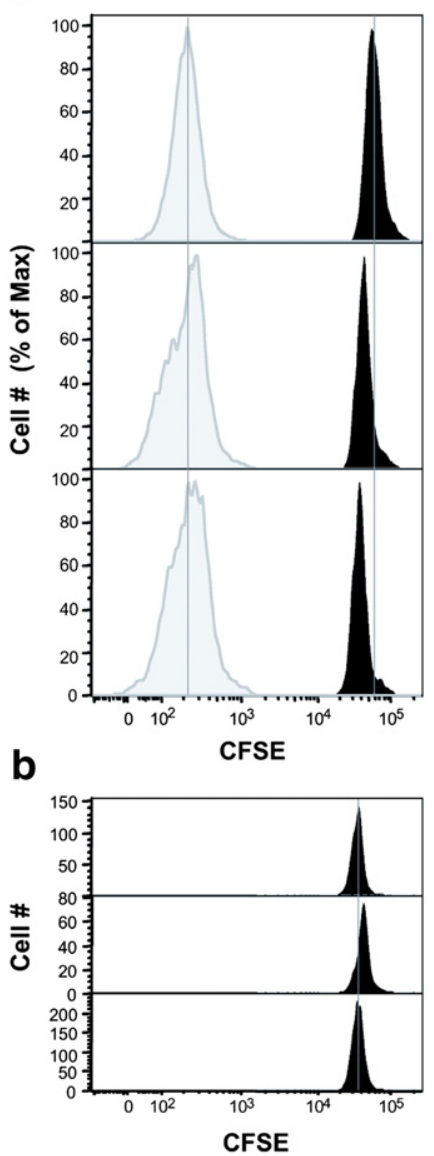

Unlabelled cells
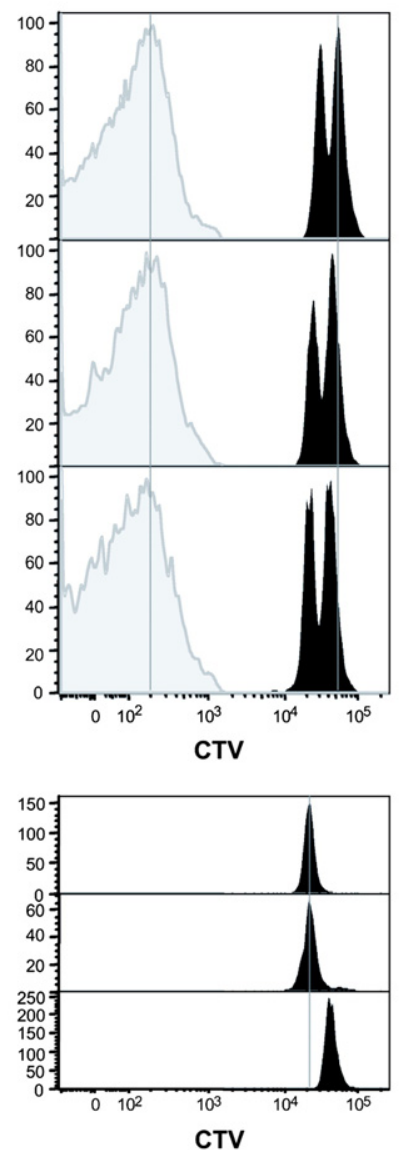

Dye-labelled cells
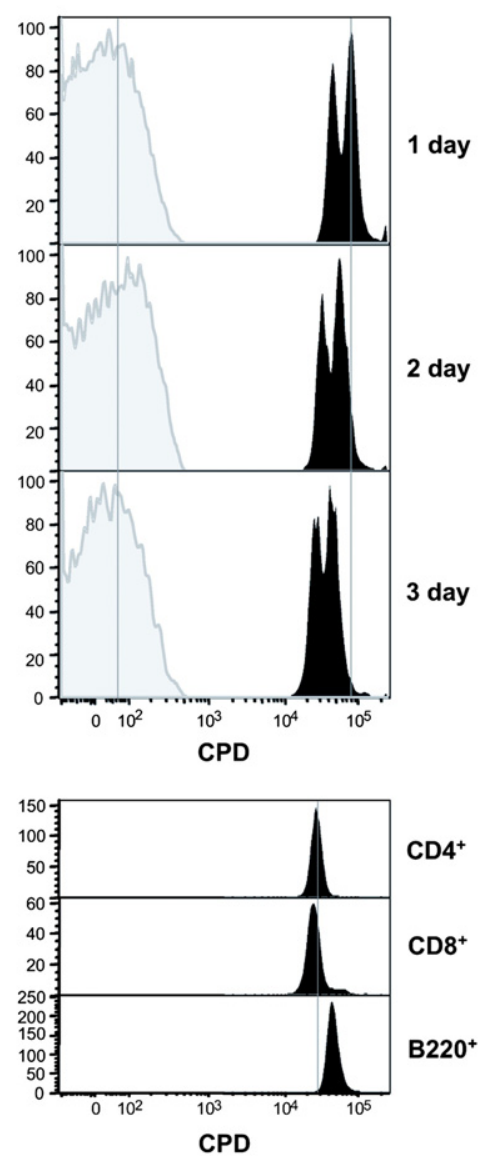

Fig. 1. High level and long lived fluorescent labelling of splenocytes by CFSE, CTV and CPD.Spleen cells from B6 mice were labelled with $10 \mu \mathrm{M}$ CFSE, CTV or CPD and cultured for 3 days. Viable (Hoechst $33258^{-}$) cells were assessed for levels of dye fluorescence at each day of incubation using identical settings on a Fortessa flow cytometer at each time point. a) Histogram plots show fluorescence of labelled cells relative to unlabelled cells. Vertical dotted lines show peak levels of fluorescence at day 1 , revealing the decay in cell fluorescence over time. b) $\mathrm{CD}^{+}{ }^{+}, \mathrm{CD}^{+}$, and B (B220 ${ }^{+}$) cells from the 3 day samples were discriminated using specific antibody staining and assessed for dye labelling intensity. Vertical dotted lines show peak levels of fluorescence of CD4 ${ }^{+}$cells, revealing that different subpopulations of cells label to different extents with the three dyes.

and B cells using subpopulation-specific antibodies (Fig. 1b). This revealed that there was some degree of variability in the labelling of these lymphocyte subsets, the most prominent being the higher labelling of B cells by CTV and CPD, which accounted for the more highly fluorescent subpopulation seen in splenocyte preparations labelled with each of these dyes. These data demonstrate the importance of discriminating lymphocyte subsets to obtain the best peak variance of all three dyes and also shows that CTV and CPD can stably label cells to a similar level of low variance fluorescence as CFSE, making them excellent dyes for cell labelling.

\subsection{CFSE, CTV and CPD detect proliferating lymphocytes to vary- ing degrees of resolution}

The capacity of the different fluorescent dyes to measure cell proliferation was tested on $\mathrm{CD}^{+}{ }^{+} \mathrm{T}$ cells, $\mathrm{CD}^{+}$ $\mathrm{T}$ cells and $\mathrm{B}$ cells by stimulating dye-labelled mouse splenocytes with a cocktail of $\mathrm{T}$ cell and $\mathrm{B}$ cell mitogens for
3 days in vitro, at which time they were analysed by flow cytometry (Fig. 2). Consistent with previous findings, CFSE was able to detect discrete proliferation peaks for the $\mathrm{CD}^{+}$and $\mathrm{CD}^{+} \mathrm{T}$ cell populations, with up to 8 peaks being detected before fluorescence levels reached the background autofluorescence of activated non-CFSE labelled cells (Fig. 2a). In contrast, although the B cell population had clearly diluted CFSE and therefore had divided, discrimination of individual proliferation peaks was very poor with the B cell population compared to the two T cell populations. Similarly, CTV was very capable of resolving $\mathrm{CD}^{+}$and $\mathrm{CD}^{+} \mathrm{T}$ cell divisions, but was poor at detecting $\mathrm{B}$ cell divisions. An obvious difference between the CFSE and CTV dyes, however, was that CTV was less capable of detecting later cell divisions. For example, with CFSE-labelled $\mathrm{CD}^{+} \mathrm{T}$ cells, 8 fluorescent peaks could be resolved, whereas with CTV only 6 peaks were clearly discernable. Of all the dyes tested, CPD was obviously far inferior in detecting cell division, with division 
a \#

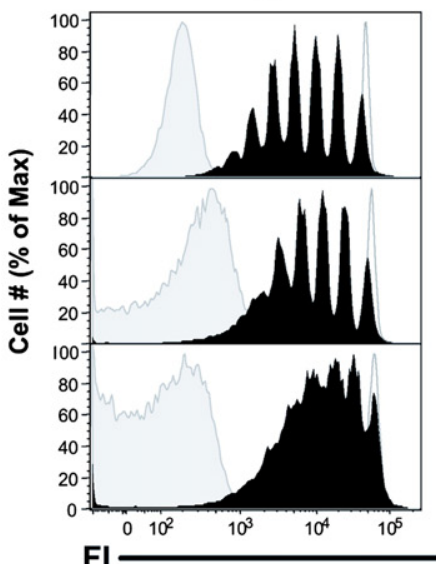

$\mathrm{CD}^{+}$

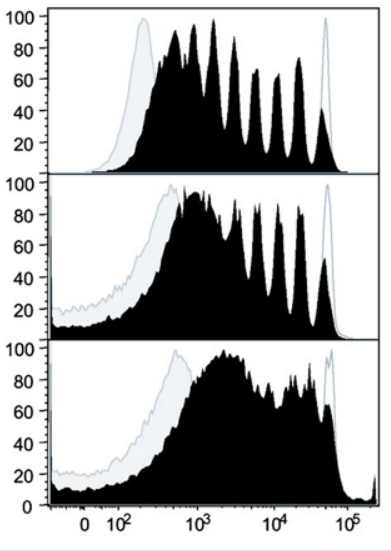

$\mathrm{B220}^{+}$

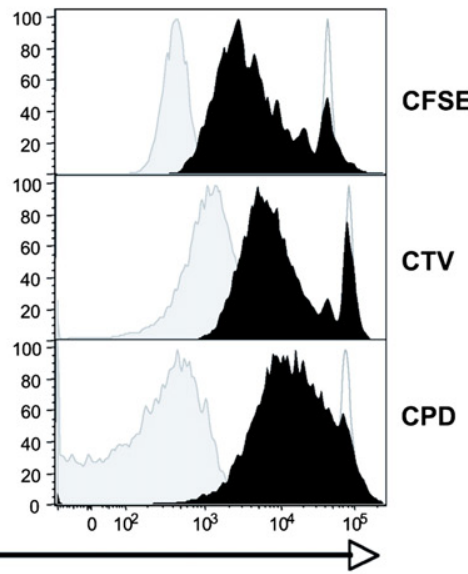

CFSE

PD

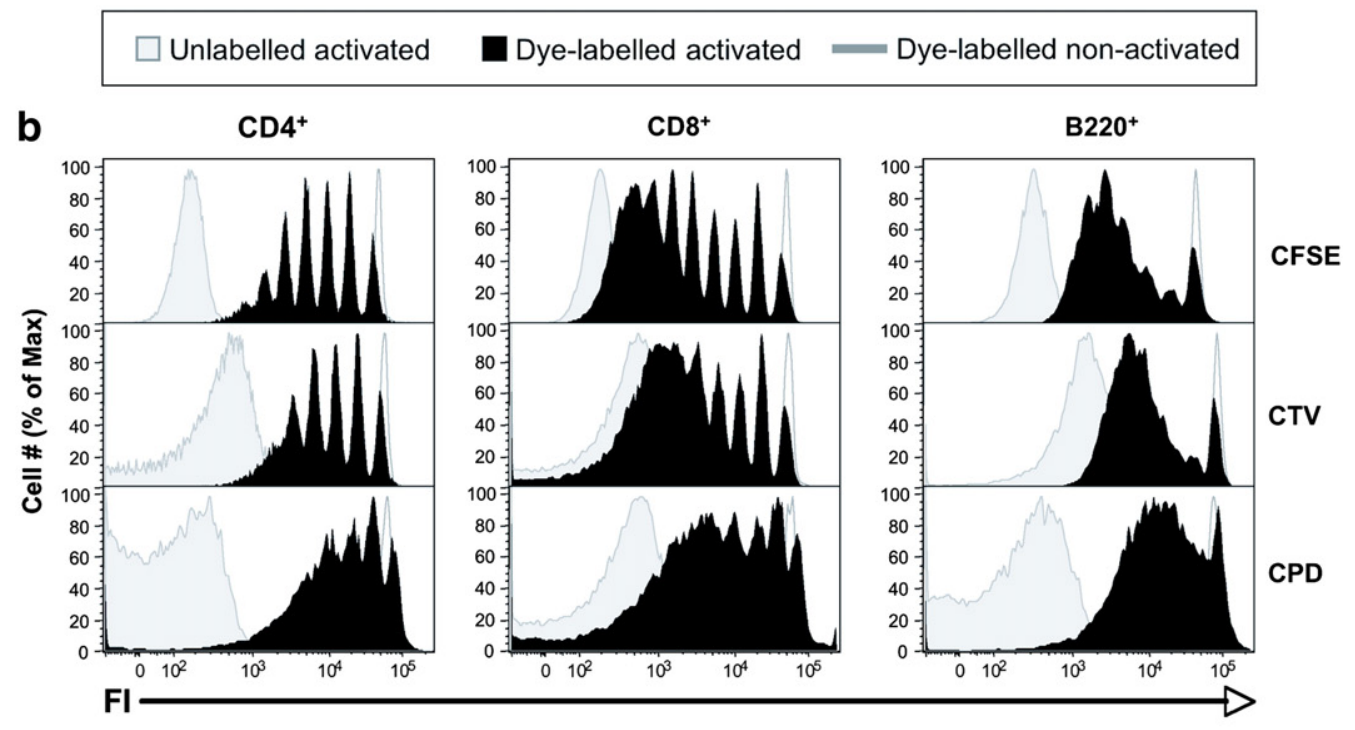

C

Live cells
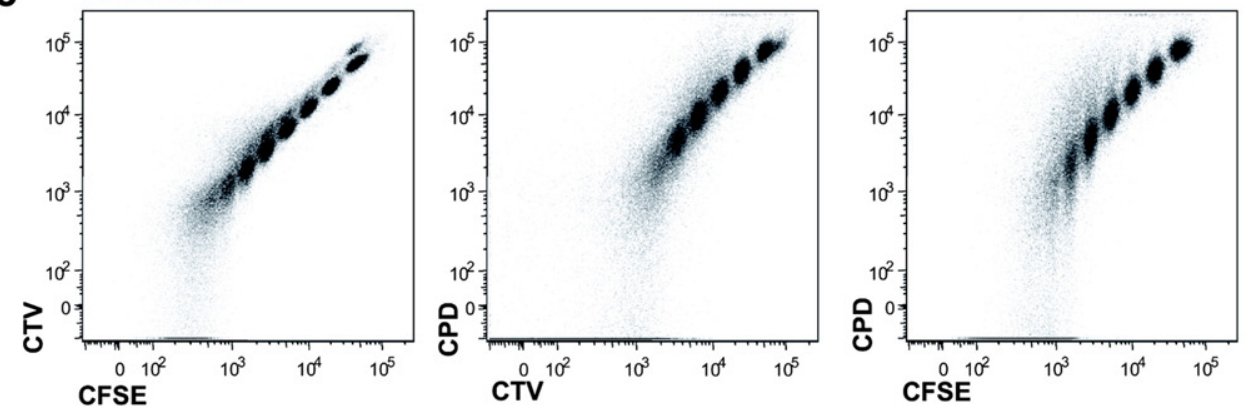

Fig. 2. Detection of lymphocyte division by CFSE, CTV and CPD in vitro.Spleen cells from B6 mice were labelled with $10 \mu \mathrm{M}$ CFSE, CTV and/or CPD and cultured for 4 days in the presence of a range of polyclonal stimuli as described in the Methodssection that activate $\mathrm{T}$ and $\mathrm{B}$ cells (6.25\% of the maximum stimulation is shown). Viable (Hoechst $33258^{-}$) $\mathrm{CD}^{+}, \mathrm{CD}^{+}$, and $\mathrm{B} 220^{+}$cells were discriminated using specific antibody staining. a) Histogram plots showing division peaks following stimulation of cell populations labelled individually with CFSE, CTV or CPD. Controls include non-activated labelled cells (non-dividing) and unlabelled cells activated by the polyclonal stimuli (background autofluorescence). FI $=$ fluorescence intensity. b) Viable lymphocyte subsets $\left(\mathrm{CD}^{+}{ }^{+}, \mathrm{CD}^{+}{ }^{+}, \mathrm{B}^{2} 20^{+}\right.$) co-labelled simultaneously with CFSE, CTV and CPD were assessed for fluorescent division peaks in histogram plots as in a). c) Activated viable cells co-labelled with all 3 fluorescent dyes were also assessed for fluorescence of each dye relative to the other two dyes in 2D density plots.

peak resolution being very poor and few peaks being discernable, this deficiency being the most extreme with proliferating B cells.
One problem encountered with the proliferation assays described above was that comparisons were made between different samples labelled with each fluorescent 
dye and so it was unclear if all three dyes were detecting cells in the same round of division. For example, in the $\mathrm{CD}^{+} \mathrm{T}$ cell population labelled with CTV, it was unclear if the cells had undergone up to 7 divisions (as with CFSE) or only 5 divisions (Fig. 2a). To overcome this problem splenocytes were labelled simultaneously with all three dyes making it possible to directly relate lymphocyte divisions detected by CFSE with the division profiles of the other two dyes. Co-labelling in this way revealed nearly identical dye division profiles as splenocytes labelled separately with the three different dyes (compare Fig. 2a and 2b). Co-labelling also provided the opportunity to construct 2-dimensional fluorescence density plots that revealed the relationship between cell divisions and the fluorescence of the different dyes (Fig. 2c). This comparison confirmed that CTV fluorescence exhibited a broader variance in later cell division peaks compared to CFSE, resulting in later divisions detected by CTV fluorescence being extremely difficult to resolve. CPD fluorescence had division peaks with broader variance compared to the fluorescence division peaks detected by both of the other dyes. Importantly all three dyes, even when used to colabel the same cells, had very little impact on cell viability and proliferation (i.e., viable cell number) relative to unlabelled cells over a wide range of stimuli strengths, demonstrating that all three fluorescent dyes had minimal toxicity for cells (Supplementary Fig. 2).

Thus, overall it appears that CTV-labelling is comparable to CFSE-labelling in measuring lymphocyte division, although CTV-labelling of cells results in less resolution of division peaks at higher dye dilutions. In contrast, CPDlabelling results in poorly defined cell division peaks. Also of note is that, compared to $\mathrm{T}$ cells, B cell division peaks are far more difficult to resolve with all three fluorescent dyes.
3.3. Resolution of division peaks by CPD and detection of B cell division by all dyes is superior in vivo

To assess how well CFSE, CTV and CPD labelling detects lymphocyte proliferation in vivo, OVA-specific (OT-I) $\mathrm{CD}^{+}$ $\mathrm{T}$ cells, OVA-specific (OT-II) $\mathrm{CD}^{+}{ }^{+} \mathrm{T}$ cells and HEL-specific (MD4) B cells were co-labelled with all three fluorescent dyes and adoptively transferred into B6.CD45.1 host mice, which were then challenged with a HEL-OVA conjugate to stimulate all three transferred lymphocytes subsets. After 3 days, host spleens were collected and adoptively transferred $\mathrm{Tg}$ lymphocytes distinguished from host lymphocytes by CD45 allotypic differences, and assessed for proliferation using the three fluorescent dyes (Fig. 3). As in the in vitro studies, CTV labelling was able to detect discrete proliferation peaks as effectively as CFSE labelling, but with slightly less resolution in the later divisions (i.e., compare data from the $\mathrm{CD}^{+}$populations). In contrast to the in vitro data, CPD labelling appeared to have a much better capacity to resolve division peaks in vivo. What was also striking was that the B cell division peaks in vivo were much more discrete than those seen in vitro when using any of the three fluorescent dyes. These differences between the in vitro and in vivo proliferation profiles did not appear to be due to the different stimulation conditions used in the in vitro and in vivo assays, since Tg lymphocytes stimulated with specific antigen or polyclonal stimuli in vitro gave similar dye dilution profiles to non-Tg cells stimulated with polyclonal mitogens in vitro (data not shown).

To quantify the differences in the lymphocyte proliferation profiles generated between the in vivo and in vitro studies and between the $\mathrm{B}$ and $\mathrm{T}$ cell subsets, two parameters were enumerated, namely, the mean fluorescence intensity (MFI) of each successive division peak (which theoretically should halve after each cell division) and division peak variance (which should be as small as possible for best peak
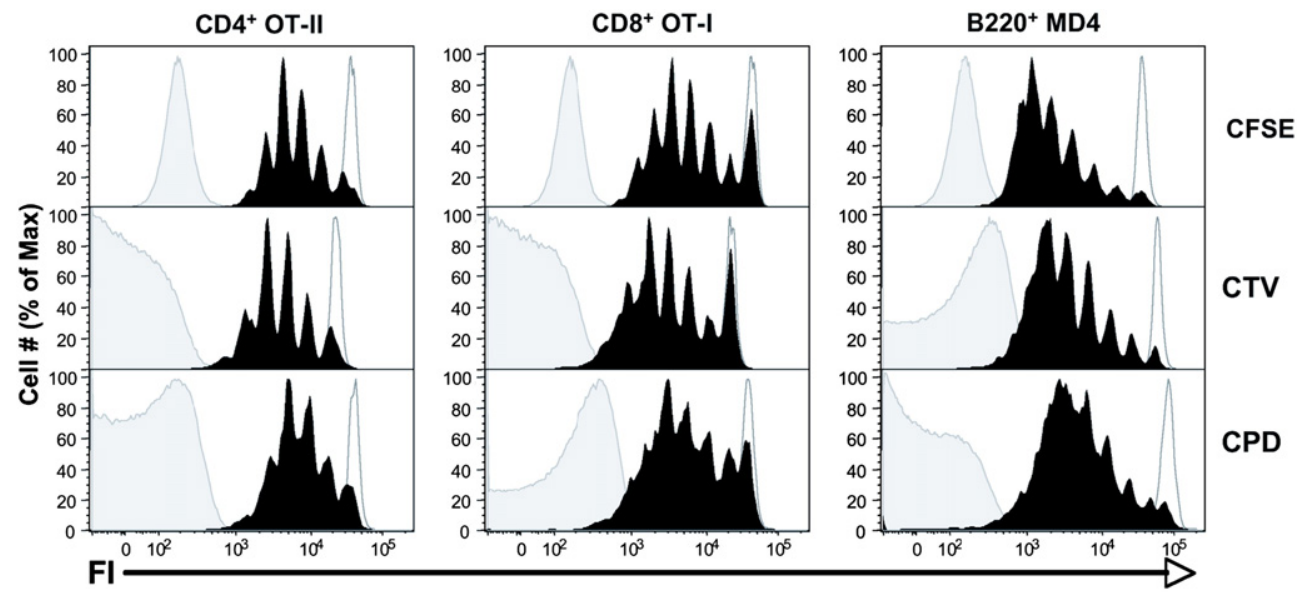

Unlabelled activated

Dye-labelled activated

Dye-labelled non-activated

Fig. 3. Detection of lymphocyte division by CFSE, CTV and CPD in vivo.CD4 ${ }^{+}$T cells from OT-II mice, CD8 ${ }^{+}$T cells from OT-I mice and B cells from MD4 mice were simultaneously labelled with $10 \mu \mathrm{M}$ CFSE, CTV and CPD and the cells adoptively transferred into host B6.CD45.1 mice. Separate host mice were challenged i. v. with $1 \mu \mathrm{g}$ of HEL-OVA for CD4 ${ }^{+}$(OT-II) T cell responses, $10 \mu \mathrm{g}$ of HEL-OVA for CD8 ${ }^{+}$(OT-I) T cell responses or $10 \mu \mathrm{g}$ of HEL-OVA together with $10 \mu \mathrm{g}$ of LPS for

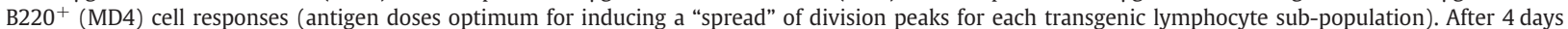

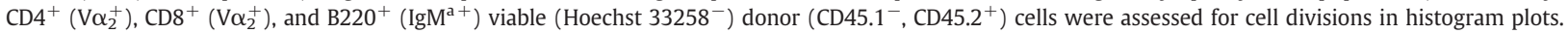
Controls include non-activated labelled cells (non-dividing) and unlabelled activated cells (background autofluorescence). FI $=$ fluorescence intensity. 
resolution). To do this proliferating lymphocytes, either in vitro or in vivo and co-labelled with all three fluorescent dyes, were delineated into each cell division (numbered 0 to 6 ) based on the fluorescence intensity of two of the fluorescent dyes and the fluorescence statistics of the third dye then generated for each cell division (Supplementary Fig. 3 ). In this way division peak fluorescence statistics could be generated for each fluorescent dye without using the fluorescence of the dye being considered in the gating strategy. Overall, the MFI of each successive division peak decreased at a constant rate (log reduction) for all three of the fluorescent dyes, irrespective of whether the lymphocytes were proliferating in vitro or in vivo (Supplementary Fig. 4). However, in vitro there appeared to be a greater retention of CPD by lymphocytes in early divisions, then a greater loss of CPD in later divisions, compared to the other dyes. These in vitro properties of CPD were more exaggerated in proliferating $\mathrm{B}$ cells than in proliferating T cells (Supplementary Fig. 4).

The variance of fluorescence of each division peak was measured by the coefficient of variance (CV), a normalised measure of variance which equals the standard deviation of a fluorescent peak as a percentage of the MFI (Fig. 4). The CV of CPD fluorescence across all division peaks and all lymphocyte subsets was greater than the CV of CFSE and CTV fluorescence, this difference being particularly evident with later cell divisions and in the B cell subset. Furthermore, this difference was more pronounced with lymphocytes proliferating in vitro. The CV of CFSE and CTV fluorescence across most division peaks was similar in T lymphocytes proliferating both in vitro and in vivo, with the exception being that with later division peaks the CV of CTV fluorescence always appeared to be greater than the CV of CFSE fluorescence. In contrast, CFSE and CTV labelled B cells proliferating in vitro had higher division peak CVs than proliferating $\mathrm{T}$ lymphocytes (Fig. 4). Generally, it appeared that if the fluorescence intensity of adjacent division peaks had a standard deviation of $\leq 20 \%$ of the MFI, division peak resolution was very good. However, if the fluorescence intensity of adjacent division peaks had a standard deviation of $\geq 30 \%$ of the MFI, division peak resolution was very poor (Fig. 4).

From this statistical analysis, it appeared that the differences in resolution of division peaks seen between the different fluorescent dyes, different lymphocyte subsets and in vivo versus in vitro proliferation assays correlated well with changes in the $\mathrm{CV}$ of the division peaks. In subsequent experiments we attempted to understand how such changes in division peak variance occurred and ultimately improve division peak resolution by optimising assay conditions.
$\mathrm{CD4}^{+}$

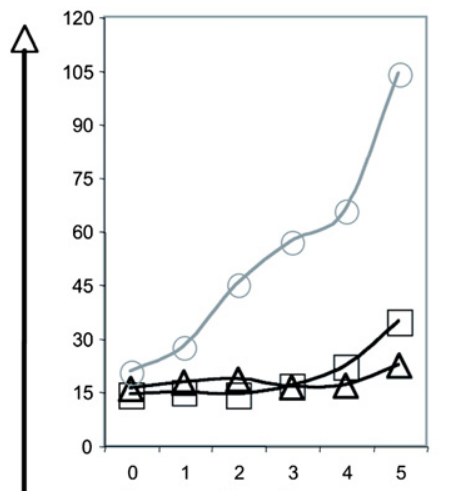

Generation \#

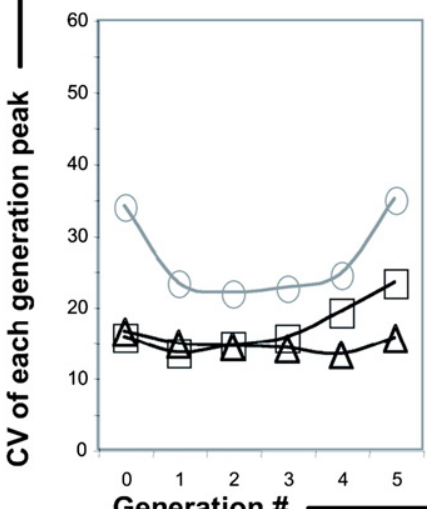

$\mathrm{CD}^{+}$
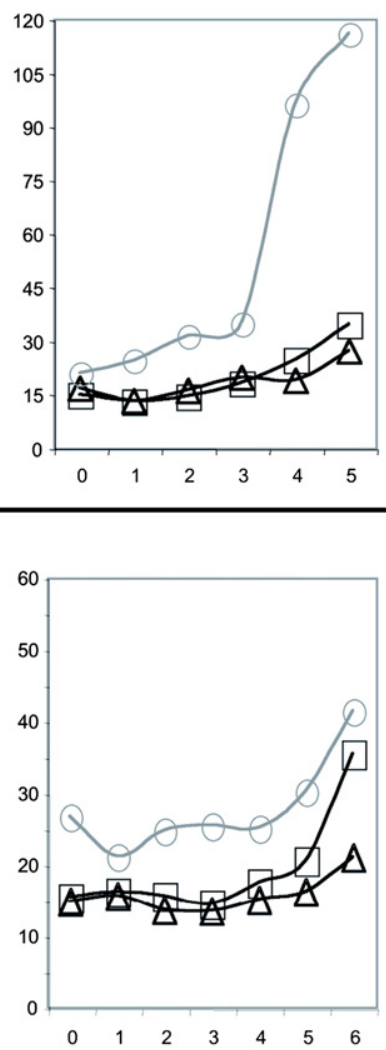

$\mathrm{B}^{2} \mathrm{O}^{+}$

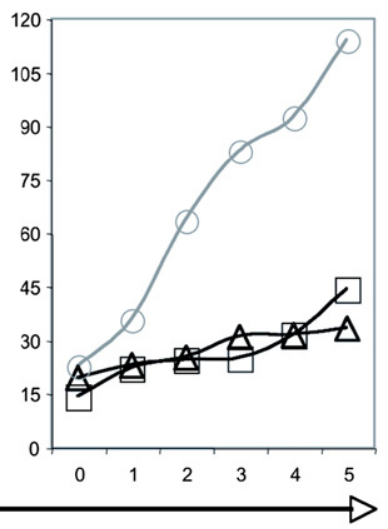

In vitro

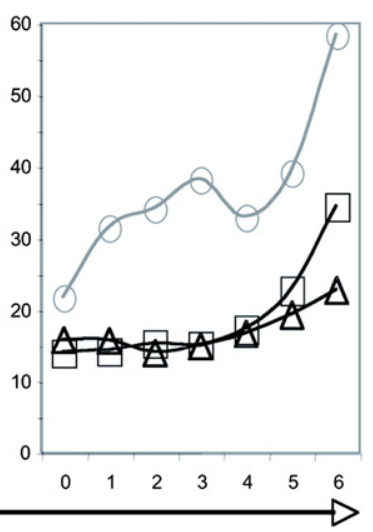

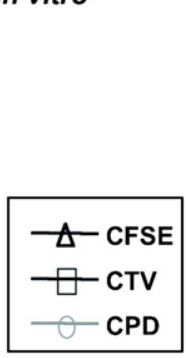

In vivo

Fig. 4. Co-efficient of variance statistics of individual division peaks for CFSE, CTV and CPD-labelled lymphocyte subsets from in vitro and in vivo assays.The coefficient of variance percentage (CV) of the individual generation peaks identified in S Fig. 3 was determined using FlowJo software and plotted against generation number. This was done for $\mathrm{CD} 4^{+}, \mathrm{CD}^{+}$and $\mathrm{B}_{2} 20^{+}$cells activated in vitro and in vivo as described in Figs. 2 and 3, respectively. 


\subsection{Role of dye transfer between lymphocytes in division peak} resolution

Initially we examined why CPD fluorescence and B cell fluorescence with all three dyes had such high variance with each division peak, and why this effect was more pronounced in lymphocytes proliferating in vitro than in vivo. A potential explanation for this high fluorescence variability is through fluorescent dye transfer between lymphocytes. For example, if CPD transferred between cells more readily than CFSE and CTV, division peak fluorescence variability would be greater with CPD labelled cells than CFSE or CTV labelled cells. Furthermore, if B cells had a greater capacity to transfer the dyes between one another than $\mathrm{T}$ cells, this might account for the poor resolution of B cell divisions seen with each of the fluorescent dyes. Dye transfer between cells might also explain why in vitro culture of dividing cells exacerbates poor division peak resolution, since dye-labelled cells would be closely associated with one another and thus able to transfer the dyes more readily than when the cells were placed in an animal. To assess dye transfer in vitro, splenocytes labelled separately with each of the fluorescent dyes were co-cultured for 3 days and dye transfer between the lymphocytes assessed by flow cytometry (Fig. 5). To assess dye transfer in vivo, splenocytes labelled with all three fluorescent dyes were transferred into host mice, and dye transfer from donor dye-labelled cells to unlabelled host cells assessed by flow cytometry (Fig. 5). Lymphocytes transferred substantial amounts of CPD and minimal amounts of CFSE and CTV when cultured in vitro (Fig. 5a, upper panels). In contrast, dye-labelled lymphocytes did not appear to transfer any of the dyes to host splenocytes in vivo (Fig. 5a, lower panel). Lymphocyte subset analysis of the in vitro proliferation assays revealed that with all three fluorescent dyes $\mathrm{B}$ cells acquired more fluorescent dye from bystander cells than $\mathrm{CD}^{+}{ }^{+}$and $\mathrm{CD}^{+} \mathrm{T}$ lymphocytes (Fig. 5b). Therefore, it appears that decreases in division peak resolution seen in lymphocyte proliferation assays correlate well with dye transfer between the cells.

\subsection{Role of lymphocyte autofluorescence in division peak resolution}

A common property of CFSE, CTV and CPD-labelled lymphocytes was a loss in division peak resolution at later cell divisions. While dye transfer may account for some of this loss in division peak resolution, it was also observed in lymphocytes from in vivo proliferation assays where dye transfer was negligible. This suggested that other factors also play a role in loss of division peak resolution at later cell divisions. One possibility is that fluorescent peak resolution may be compromised when the fluorescence of dividing cells approaches that of cell autofluorescence. Two parameters are important when considering the effects of cell autofluorescence on division peak resolution, namely, (i) the number of divisions permissible before dye fluorescence intensity reaches background autofluorescence levels (i.e., the MFI of autofluorescent cells) and (ii) the variance of the autofluorescent cells (i.e., the variance that dye-labelled cells might adopt as they approach cell autofluorescence levels). Indeed, the autofluorescence variance of lymphocytes in the three channels used to detect each of the fluorescent dyes was much greater than the fluorescence variance of the dyelabelled lymphocyte division peaks (Fig. 6a). Furthermore, the autofluorescence variance of lymphocytes, as measured by $C V$, was larger in the CPD-channel than in the CTV-channel, which in turn was larger than the autofluorescence variance of lymphocytes in the CFSE channel. This was observed across all lymphocyte subsets and concentrations of mitogens used (Fig. 6b, upper panel). These trends correlate well with the decreased resolution of late division peaks seen with all three fluorescent dyes, with CPD fluorescence being worse than CTV and CTV fluorescence being worse than CFSE. This may explain why CTV labelled cells exhibited poorer resolution of late cell divisions than CFSE labelled cells, despite CTV displaying lower dye transfer between lymphocytes. It is also important to note that the mean autofluorescence intensity of lymphocytes in the CTV channel increased as the cells were activated and this increase occurred more rapidly than in the CFSE channel (particularly in the case of B cells) (Fig. 6b, lower panel). Thus the capacity of CTV labelling to detect later cell divisions is compromised due to both the increased autofluorescence intensity of activated cells and the higher levels of autofluorescence variance that lymphocytes intrinsically possess in the CTV channel. Cells in the CFSE channel are not as greatly affected by these parameters.

\subsection{Enhancing division peak resolution}

From these studies it appears there are two potential ways to increase the resolution of lymphocyte division peaks detectable by fluorescent dyes, namely decreasing intercellular dye transfer and/or decreasing the effects of cell autofluorescence. While it is difficult to alter the capacity of cells to transfer dyes to bystander cells, decreasing the effect of cell autofluorescence might be achieved by increasing the MFI of labelled lymphocytes such that it is much higher than autofluorescence levels. To examine whether this approach was feasible, lymphocytes were labelled with double (i.e., $20 \mu \mathrm{M}$ ) and quadruple (i.e., $40 \mu \mathrm{M}$ ) the normal concentration of the fluorescent dyes and lymphocyte proliferation assessed (Fig. 7). Increasing the dye concentration did indeed result in an increase in the resolution of later cell division peaks. This was particularly obvious with $\mathrm{T}$ lymphocytes labelled with CFSE and CTV where, for example, CD8 ${ }^{+} \mathrm{T}$ cells labelled with higher concentrations of each dye had progressively better resolution of the 7th and 8th dye peak, these peaks being completely merged together when using the $10 \mu \mathrm{M}$ concentration of each of the dyes. In contrast, there was only a modest increase in resolution of later cell division peaks with $B$ cells labelled with higher concentrations of the dyes or all lymphocyte subsets labelled with higher concentrations of CPD (Fig. 7). Notably, viability was not compromised when lymphocytes were labelled with these higher dye concentrations, even when lymphocytes were simultaneously labelled with $40 \mu \mathrm{M}$ of all three fluorescent dyes (i.e., $120 \mu \mathrm{M}$ total dye concentration) (Supplementary Fig. 5). It 
a

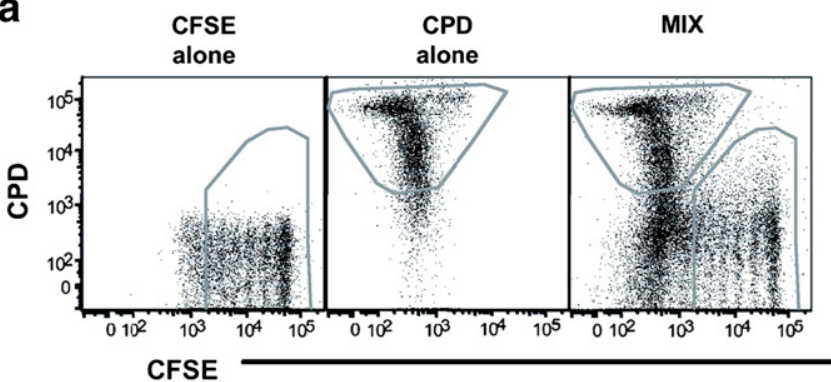

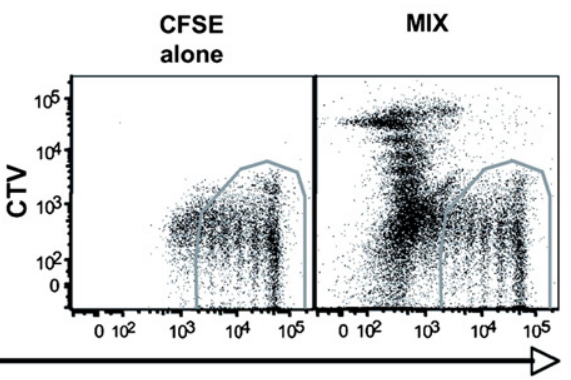

\section{$\mathbf{3}$}

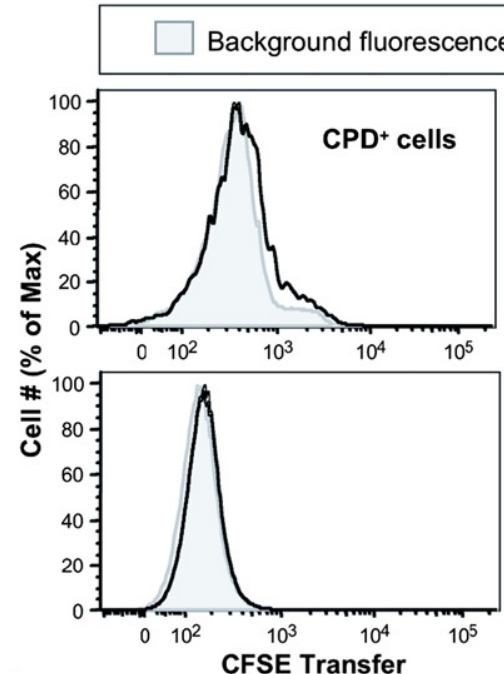

b

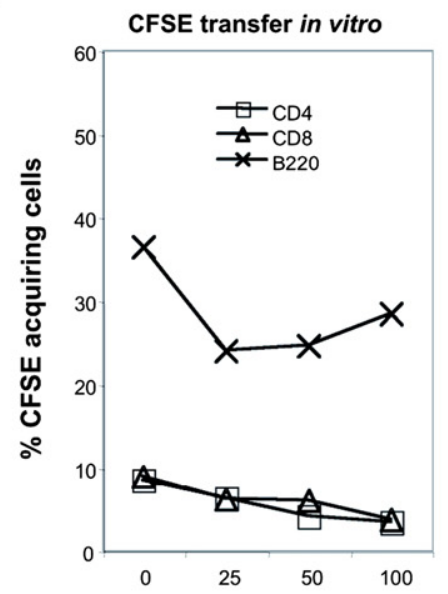

Fluorescence in the presence of dye-labelled donor cells
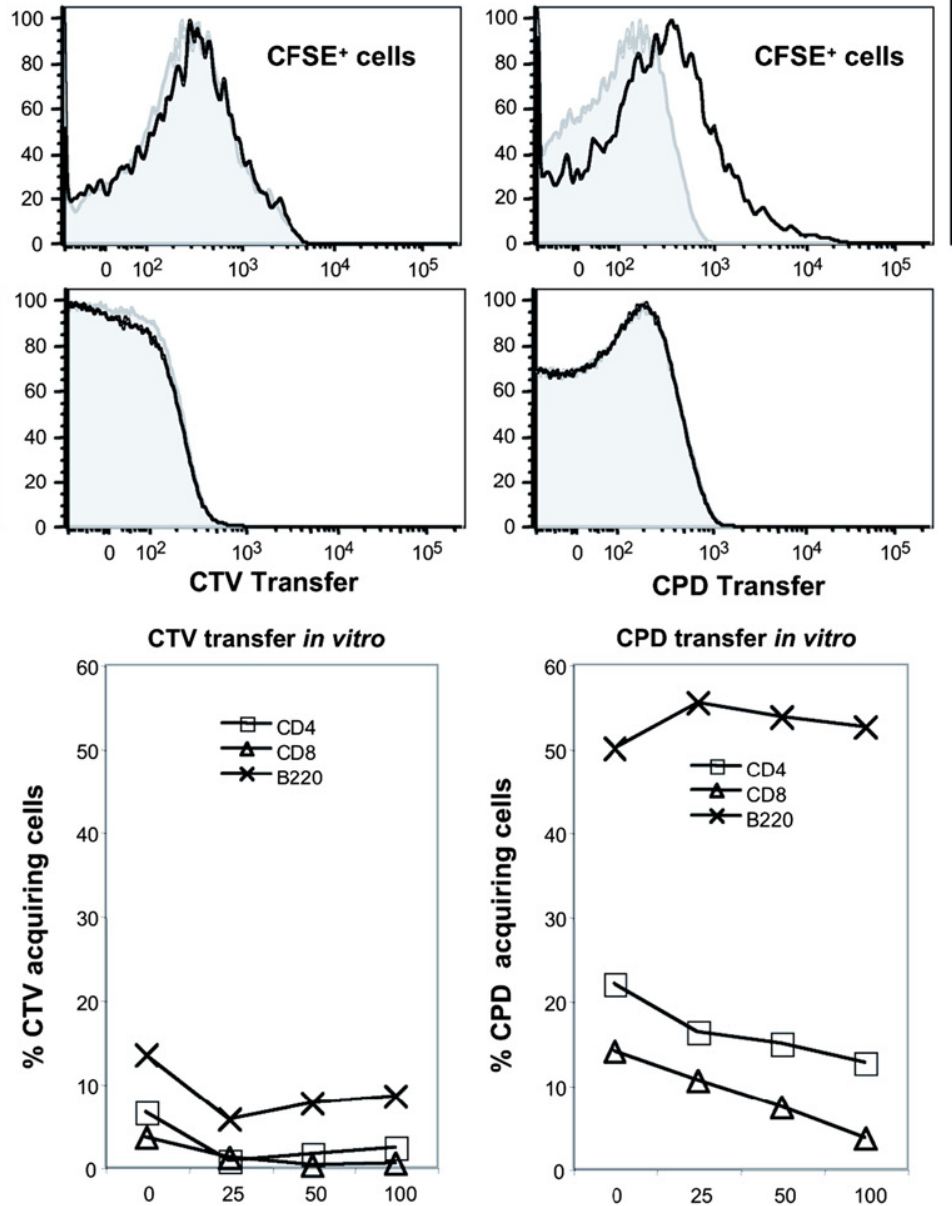

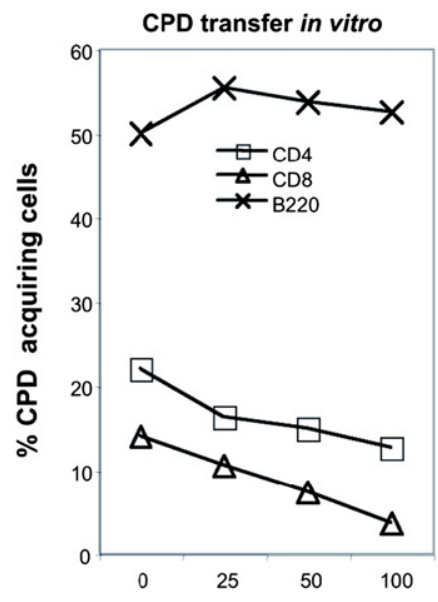

Stimuli $(\%)$

Fig. 5. Ability of the different fluorescent dyes to transfer to bystander lymphocytes.a) Lymphocytes labelled individually with CFSE, CTV or CPD were cultured alone or together and activated in vitro as described in Fig. 2. After 3 days of culture, transfer of each dye to bystander cells was assessed by comparing the dye fluorescence of cells cultured alone to those cultured together, as shown in the dot plots (top panels). Gates on the 2D dot plots were used to generate histogram plots showing the amount of fluorescent dye transfer in vitro. In addition, lymphocytes simultaneously labelled with CFSE, CTV and CPD were activated in vivo as described in Fig. 3 and the amount of fluorescent dye transfer to host (CD45.1 ${ }^{+}, \mathrm{CD} 45.2^{-}$, Hoechst $\left.33258^{-}\right)$cells assessed by comparing host animals receiving dye labelled cells to those not receiving the labelled cells (in vivo panel). b) In vitro samples as described in a) were further delineated into CD4 ${ }^{+}$, CD8 ${ }^{+}$ and $\mathrm{B} 220^{+}$subpopulations using specific antibody staining and \% cells acquiring dye assessed by Overton subtraction of histograms generated in the same manner as those in a) and these data plotted against stimuli concentration.

is important to emphasise that the use of tissue culture medium (RPMI 1640) and the presence of buffering proteins (i.e., 10\% FCS) in the labelling solution is essential to prevent cellular toxicity. The new labelling conditions are based on previous studies showing that the presence of protein can reduce the toxicity of CFSE for cells (Quah et al., 2007).

An added benefit of using higher concentrations of the dyes to label cells is that it is possible to detect $>7$ cell 
a

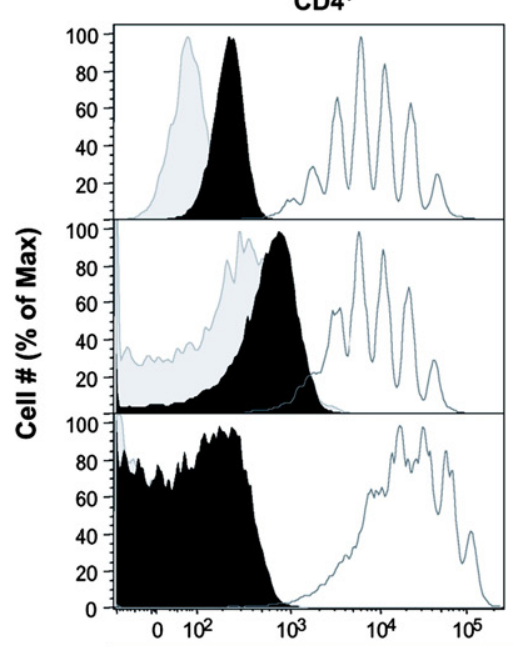

$\mathrm{CD}^{+}$

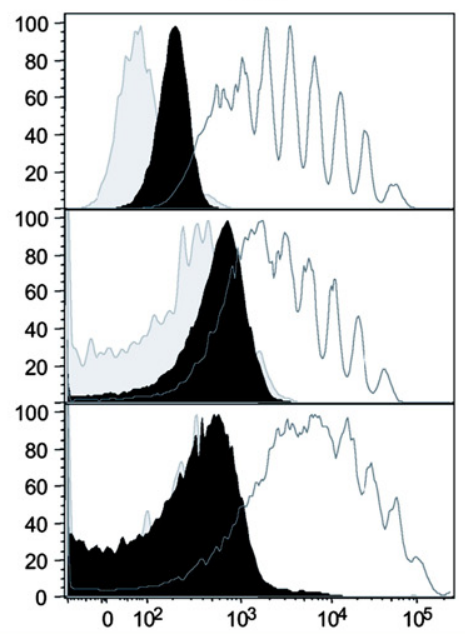

$\mathrm{B220}^{+}$

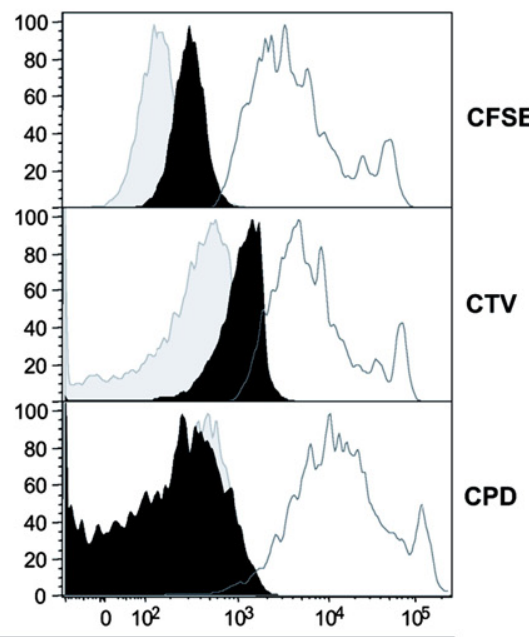

b
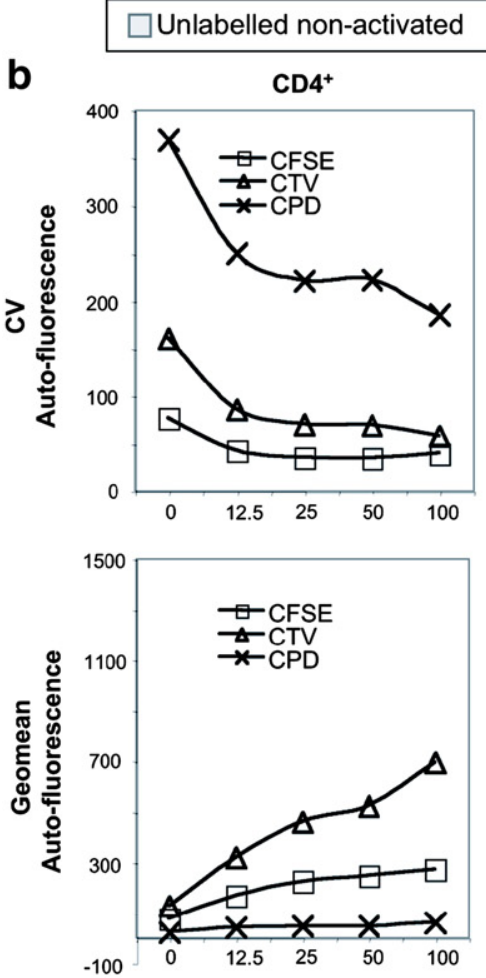

Unlabelled activated
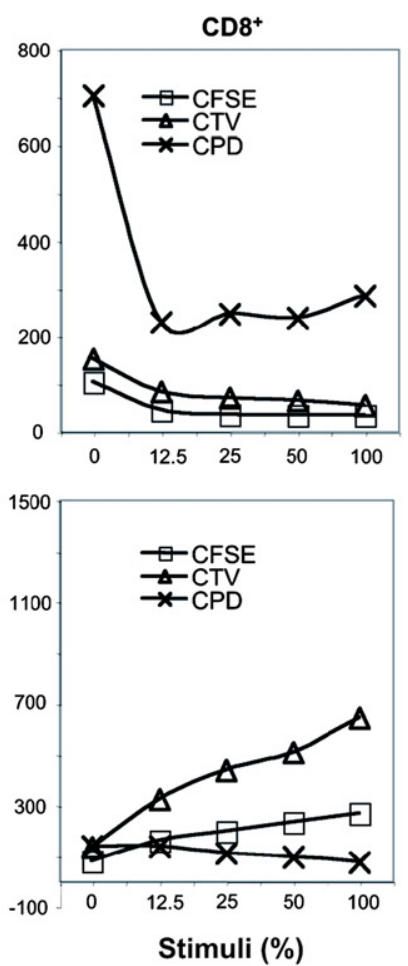

Dye-labelled activated
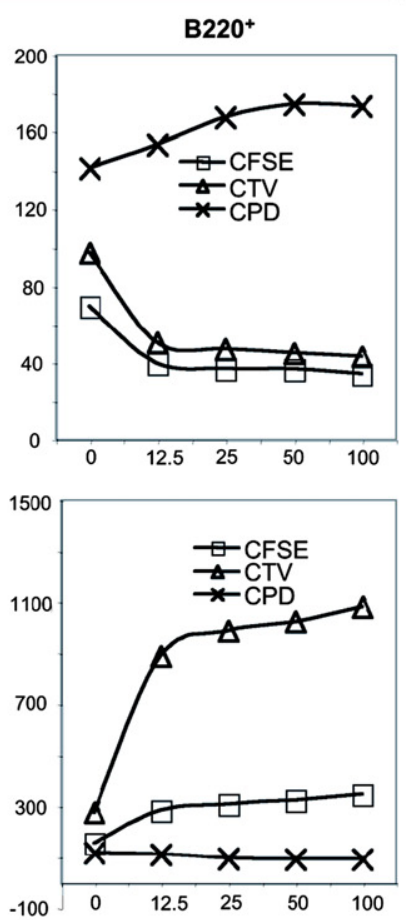

Fig. 6. Analysis of the autofluorescence of activated and non-activated lymphocyte subsets present in proliferation assays using CFSE, CTV and CPD.Lymphocytes unlabelled or simultaneously labelled with CFSE, CTV and CPD were activated in vitro as described in Fig. 2 . After 4 days, non-dye labelled viable CD4 ${ }^{+}$, CD8 ${ }^{+}$and $\mathrm{B}_{2} 2 \mathrm{O}^{+}$cells, activated or non-activated, were assessed for autofluorescence compared to activated, dye labelled cells. a) Histogram plots showing autofluorescence of different cell subsets relative to fluorescence of proliferating dye-labelled cells. b) Non-dye labelled cell subsets were assessed for autofluorescence variance (CV) and geometric mean (geomean) values plotted against stimuli concentration.

divisions, which is the maximum number achievable with CFSE using previously published labelling methods. To highlight this point, $\mathrm{CD}^{+} \mathrm{T}$ cells from OVA-specific (OT-I), NP68-specific (F5) and GP33-specific (P14.CD45.1) TCR Tg mice were labelled with $80 \mu \mathrm{M}$ of CFSE or CTV and lymphocyte proliferation assessed in an in vivo proliferation assay
(Fig. 8). After 4 days in vivo, each population of transferred $\mathrm{CD}^{+} \mathrm{T}$ cells was delineated using a combination of MHC-I tetramer binding and antibody staining and assessed for CFSE and CTV fluorescence intensity. Up to 9 fluorescent peaks were distinguishable with T cells labelled with either CFSE or CTV using this concentration of dye (Fig. 8a). 


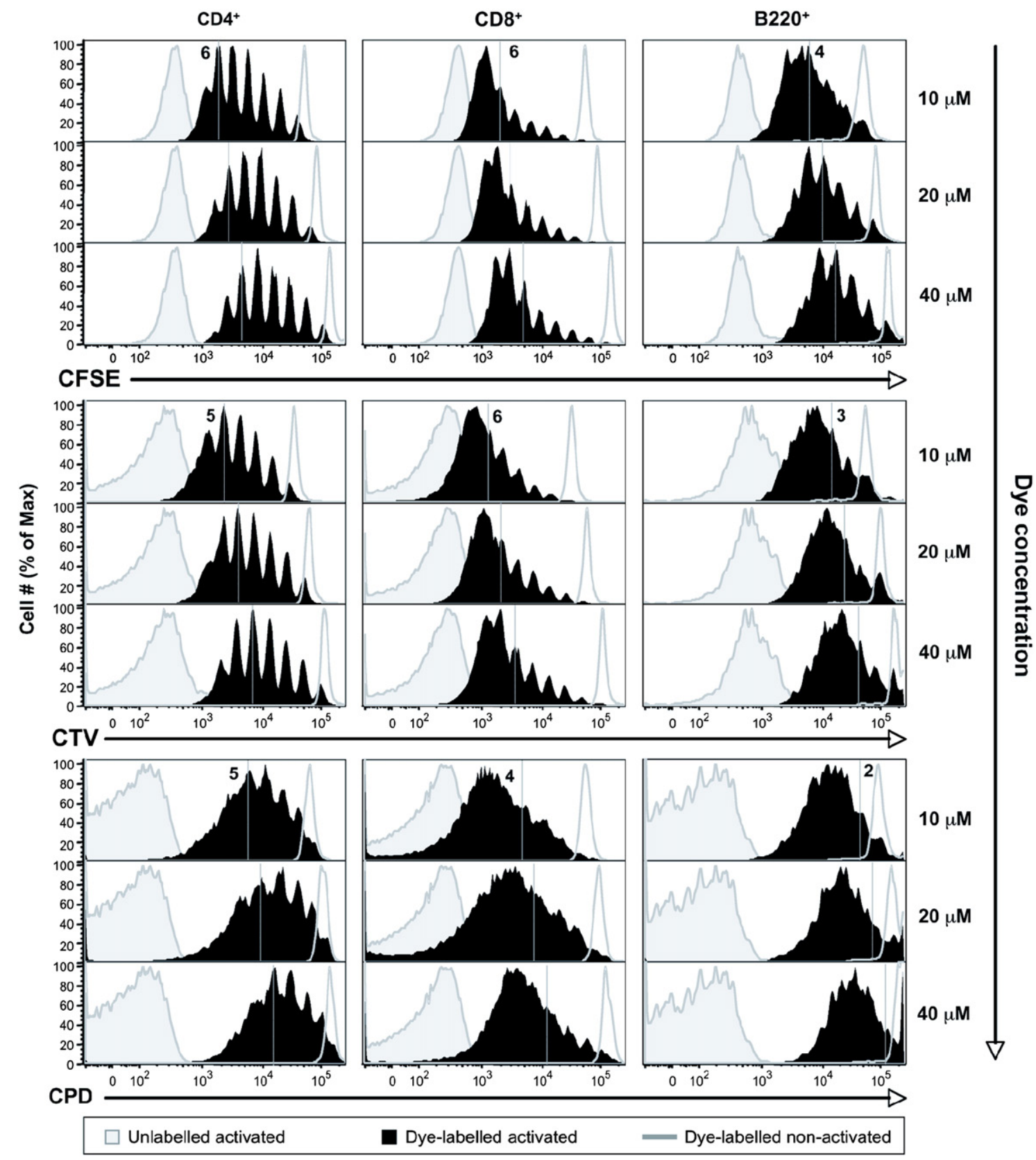

Fig. 7. The effect of labelling lymphocytes with increasing concentrations of CFSE, CTV and CPD on cell division resolution.Spleen cells from B6 mice were labelled with $10 \mu \mathrm{M}, 20 \mu \mathrm{M}$ or $40 \mu \mathrm{M}$ CFSE, CTV or CPD, activated in vitro for 3 days by polyclonal stimuli, and viable lymphocyte subsets assessed for cell division by histogram analysis as in Fig. 2. Vertical dotted lines and adjacent numbers in each set of plots represent the second last discernable division peak present in each subset with cells labelled with $10 \mu \mathrm{M}$ of each dye, this reference division peak line is also shown in histograms of cell labelled with higher concentrations of the dyes.

Additional fluorescent peaks could be visualised (up to 12 peaks and 11 cell divisions) in other experiments (Fig. 8b).

\section{Discussion}

The use of CFSE as a cell migration and proliferationmeasuring dye has had a great impact on immunological studies. Its capacity to label lymphocytes with a high level of long-lived fluorescence of low variance and low toxicity has not been replicated by any other dye for the last 20 years. Here we show that two new dyes, CTV and CPD, can also label lymphocytes with a high level of long-lived fluorescence of low variance and low toxicity making them ideal for cell tracking studies. Despite this, CPD clearly has less capacity to be retained by proliferating cells than both CFSE and CTV and this limits its application in highresolution proliferation analyses. In contrast, CTV appears to have much better retention characteristics than CPD and 
$\square$ Unlabelled activated Dye-labelled activated Dye-labelled non-activated

$\begin{array}{llllllllllll}12 & 11 & 10 & 9 & 8 & 7 & 6 & 5 & 4 & 3 & 2 & 1\end{array}$ $\begin{array}{lllllllllll}11 & 10 & 9 & 8 & 7 & 6 & 5 & 4 & 3 & 2 & 1\end{array}$
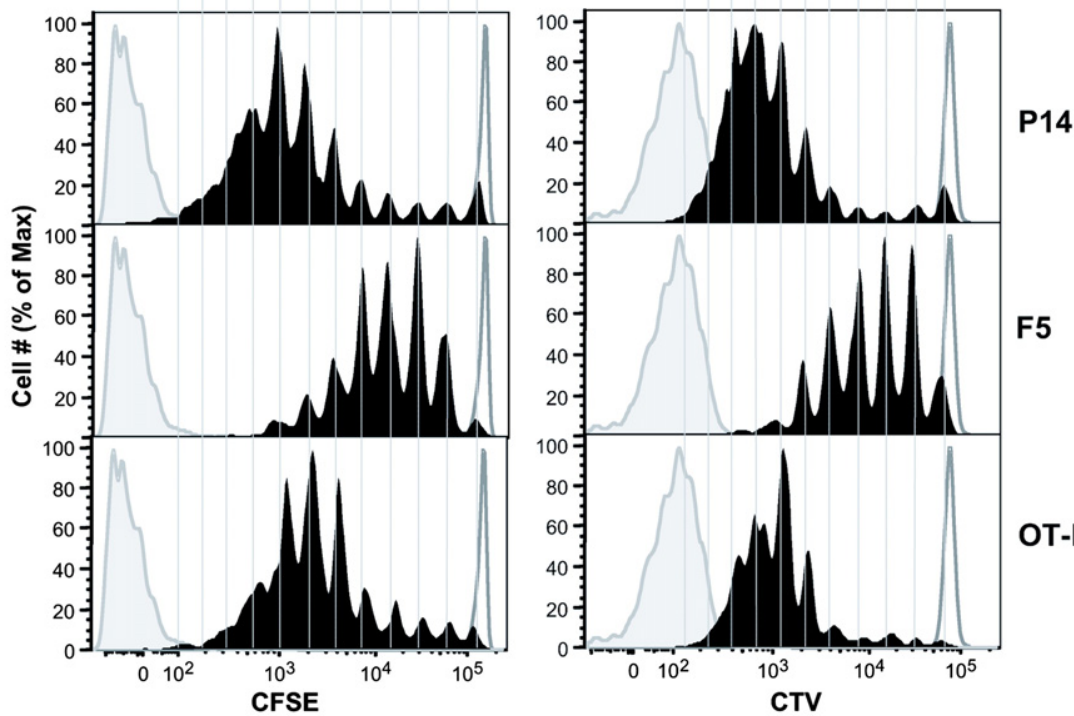

P14

OT-I
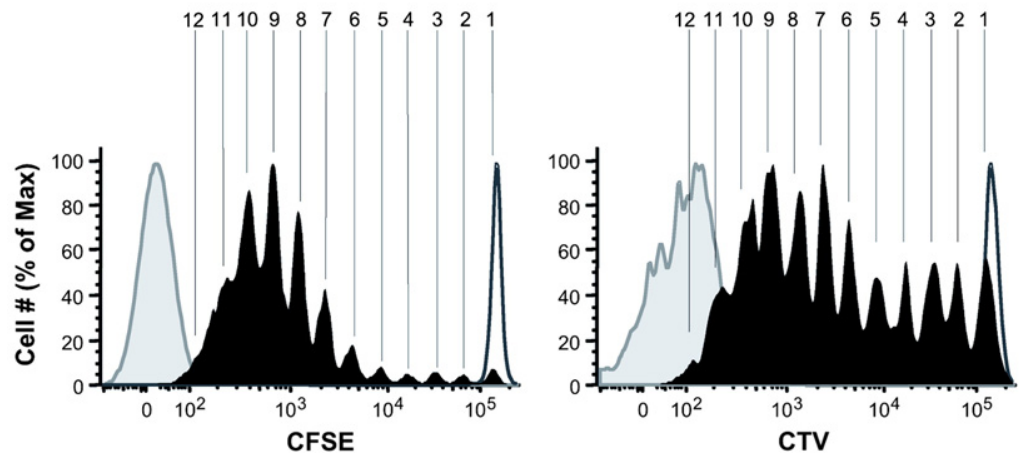

Fig. 8. Increasing the number of division peaks detectable in vivo with CFSE and CTV labelled TCR transgenic CD8 ${ }^{+} \mathrm{T}$ cells.CD8 ${ }^{+} \mathrm{T}$ cells purified from P14, F5 and OT-I TCR-Tg mice were labelled with either $80 \mu \mathrm{M}$ CFSE or $80 \mu \mathrm{M}$ CTV. The different CFSE and CTV labelled CD8 ${ }^{+} \mathrm{T}$ cells were adoptively transferred into the same host B6.CD45.1 mice and the host mice challenged with $10 \mu \mathrm{mol}$ of $\mathrm{GP}_{33-41}$ peptide (P14 antigen), $50 \mu \mathrm{mol}$ of NP ${ }_{366-374}$ peptide (F5 antigen), $25 \mu \mathrm{g}$ of OVA (OT-I antigen), and $1 \mu$ g of LPS. a) After 3 days, transferred donor CD8 ${ }^{+} \mathrm{T}$ cells were delineated using MHC-I tetramers specific for each TCR-Tg population and antibodies to CD45.1, CD45.2, $\mathrm{VB}_{11}, \mathrm{~V} \alpha_{2}$, and CD8. Donor CD8 ${ }^{+} \mathrm{T}$ cells were then assessed for proliferation using CFSE and CTV. Numbers represent division peaks. b) An example of numerous division peaks detected with CFSE-labelled P14.CD45.1 CD8 ${ }^{+}$T cells or CTV-labelled OT-I CD8 ${ }^{+} \mathrm{T}$ cells.

can resolve cell divisions to a comparable level as CFSE. Thus, CTV provides a true alternative to CFSE for measuring cell division and, due to its different spectral properties, has the advantage of being compatible with fluorochromes detected in the fluorescein channel. CTV labelling and CFSE labelling also allows the proliferation of two different populations of lymphocytes to be simultaneously measured.

While this study was mainly aimed at assessing the capacity of the dyes CTV and CPD to measure lymphocyte proliferation, it revealed some interesting details about the behaviour of CFSE-like dyes. The most obvious was the finding that fluorescent dye transfer between cells can have a severe impact on division resolution. Particularly striking was the fact that B cells appear to acquire all three fluorescent dyes tested from labelled bystander cells more readily than T cells, resulting in them having much higher variability in fluorescent dye content when dividing. This contributes to the poor resolution of division peaks with B cells in in vitro proliferation assays. This phenomenon may also explain why in vivo assays have better resolution of division peaks than in vitro proliferation assays, since acquisition of dye from nearby cells is minimised due to most adjacent cells being unlabelled.

This study also suggests that cell autofluorescence can affect cell division peak resolution as the fluorescence of dye-labelled cells approaches cell autofluorescence levels. In this regard, the autofluorescence variance of cells in the channel used to detect CTV fluorescence is generally larger than in the channel used to detect CFSE fluorescence, this effect appearing to play a role in CTV labelled cells having poorer resolution of later cell division peaks than CFSE labelled cells. Furthermore, following cell activation the mean autofluorescence of cells in the CTV channel increases relatively more than the autofluorescence of cells in the CFSE and CPD channels. This decreases the capacity of CTV labelling to detect later cell divisions. Despite this drawback the 
manufacturer of CTV claims that CTV labelling is able to resolve up to 8-10 cell divisions (Molecular Probes, Invitrogen; Applied Biosystems, Life Technologies). We could not achieve this level of division resolutions using standard concentrations of CTV for cell labelling (i.e., $10 \mu \mathrm{M}$ ). This difference may in part be due to the flow cytometers used in our study, which lack acoustic focusing, an attribute of some flow cytometers which is purported to decrease population variance and which was used for CTV analysis by the CTV manufacturers (Applied Biosystems, Life Technologies). Since many currently used flow cytometers are not be equipped with acoustic focusing, alternative approaches to increasing resolution of late division peaks are required. We have found that such increased resolution can be achieved by simply increasing the initial labelling concentration of the dyes from $10 \mu \mathrm{M}$ to $20-80 \mu \mathrm{M}$. This makes the fluorescence intensity of the labelled cells much higher than cell autofluorescence and, consequently, increases the number of proliferation peaks that can be resolved before autofluorescence interference becomes an issue. A potential problem associated with increasing the dye labelling concentration is higher fluorescence spill-over into adjacent flow cytometer detectors. The fluorescence spill-over is particularly an issue for CFSE, since CFSE fluorescence "bleeds" significantly into the PE detector and a significant amount of compensation is required to correct for this. Indeed, we found that analysis of lymphocytes labelled with $40-80 \mu \mathrm{M}$ of CFSE is difficult during the first 2 days of in vitro culture due to fluorescence spill-over (data not shown). In this respect, an advantage of CTV over CFSE is that its fluorescence has lower spectral spill-over into adjacent detectors commonly used with the violet laser line (namely the Pacific Orange detector).

Another potential constraint in using higher dye concentrations is cell toxicity. We have previously found this to be a problem even when using $2.5 \mu \mathrm{M}$ CFSE to label low concentrations of cells (Quah et al., 2007). This problem is very simply resolved, however, by labelling lymphocytes in tissue culture medium (RPMI 1640) and including a high concentration of buffering proteins (10\% FCS) in the labelling solution (Quah et al., 2007). While this slightly increases the concentration of dye needed to label lymphocytes to a certain fluorescence intensity level, the cells are able to cope with much higher concentrations of all three fluorescent dyes. Indeed, in this study we were able to use a total dye concentration of up to $120 \mu \mathrm{M}(40 \mu \mathrm{M}$ of each fluorescent dye) to label cells without a significant impact on cell viability or proliferation. This suggests that the toxicity of these dyes may be the result of excessive substitution of cell surface rather than intracellular proteins, since we anticipate that the buffering proteins would have an effect extracellularly, rather than intracellularly, during the brief labelling procedure.

Labelling lymphocytes with higher dye concentrations also allowed the detection of up to 11 cell divisions with CFSE and CTV labelled cells. To achieve these results we would recommend using $80 \mu \mathrm{M}$ of each fluorescent dye to label cells, with cell labelling being performed in tissue culture medium containing buffering proteins (in this case $10 \%$ FCS) to reduce toxicity. CFSE was originally developed as a fluorescent dye for lymphocyte migration studies (Weston and Parish, 1990) and was subsequently used to monitor lymphocyte proliferation (Lyons and Parish, 1994). The studies reported here indicate that CTV and CPD would, like CFSE, be ideally suited for labelling leukocytes for short term and long term tracking studies in vivo and, potentially, could be used to simultaneously track the migration and localisation of three different cell populations. Furthermore, the improved labelling procedure outlined in this manuscript would provide ultra-bright fluorescent leukocytes, with the CFSE-labelled cells being at least 8 times brighter than in earlier studies. Thus, despite some dye-specific limitations, CTV and CPD represent important additions to the repertoire of fluorescent dyes suitable for lymphocyte proliferation and migration experiments.

Supplementary materials related to this article can be found online at doi:10.1016/j.jim.2012.02.012.

\section{Acknowledgements}

This work was supported by a Project grant to BQ and CP from the National Health and Medical Research Council (NHMRC) of Australia. We wish to thank Harpreet Vohra and Michael Devoy for their excellent maintenance of the JCSMR FACS laboratory, and Kerong Khang and Cameron McCrae of the Australian Cancer Research Foundation Biomolecular Resource Facility, JCSMR, ANU, for the construction of MHC-I tetramers.

\section{References}

Barnden, M.J., Allison, J., Heath, W.R., Carbone, F.R., 1998. Defective TCR expression in transgenic mice constructed using cDNA-based alpha- and beta-chain genes under the control of heterologous regulatory elements. Immunol. Cell Biol. 76, 34.

Bird, J.J., Brown, D.R., Mullen, A.C., Moskowitz, N.H., Mahowald, M.A., Sider J.R., Gajewski, T.F., Wang, C.R., Reiner, S.L., 1998. Helper T cell differentiation is controlled by the cell cycle. Immunity 9, 229.

Gett, A.V., Hodgkin, P.D., 1998. Cell division regulates the T cell cytokine repertoire, revealing a mechanism underlying immune class regulation. Proc. Natl. Acad. Sci. U. S. A. 95, 9488

Goodnow, C.C., Crosbie, J., Adelstein, S., Lavoie, T.B., Smith-Gill, S.J., Brink, R.A., Pritchard-Briscoe, H., Wotherspoon, J.S., Loblay, R.H., Raphael, K., et al., 1988. Altered immunoglobulin expression and functional silencing of self-reactive B lymphocytes in transgenic mice. Nature 334, 676.

Hodgkin, P.D., Lee, J.H., Lyons, A.B., 1996. B cell differentiation and isotype switching is related to division cycle number. J. Exp. Med. 184, 277.

Hogquist, K.A., Jameson, S.C., Heath, W.R., Howard, J.L., Bevan, M.J., Carbone, F.R., 1994. T cell receptor antagonist peptides induce positive selection. Cell 76, 17.

Jedema, I., van der Werff, N.M., Barge, R.M., Willemze, R., Falkenburg, J.H., 2004. New CFSE-based assay to determine susceptibility to lysis by cytotoxic $\mathrm{T}$ cells of leukemic precursor cells within a heterogeneous target cell population. Blood 103, 2677.

Kurts, C., Kosaka, H., Carbone, F.R., Miller, J.F., Heath, W.R., 1997. Class Irestricted cross-presentation of exogenous self-antigens leads to deletion of autoreactive CD8(+) T cells. J. Exp. Med. 186, 239.

Last'ovicka, J., Budinsky, V., Spisek, R., Bartunkova, J., 2009. Assessment of lymphocyte proliferation: CFSE kills dividing cells and modulates expression of activation markers. Cell. Immunol. 256, 79.

Lyons, A.B., Parish, C.R., 1994. Determination of lymphocyte division by flow cytometry. J. Immunol. Methods 171, 131.

Mamalaki, C., Elliott, J., Norton, T., Yannoutsos, N., Townsend, A.R., Chandler, P., Simpson, E., Kioussis, D., 1993. Positive and negative selection in transgenic mice expressing a T-cell receptor specific for influenza nucleoprotein and endogenous superantigen. Dev. Immunol. 3, 159.

Marzo, A.L., Kinnear, B.F., Lake, R.A., Frelinger, J.J., Collins, E.J., Robinson, B.W., Scott, B., 2000. Tumor-specific CD4 + T cells have a major "post-licensing" role in CTL mediated anti-tumor immunity. J. Immunol. 165, 6047.

Nordon, R.E., Nakamura, M., Ramirez, C., Odell, R., 1999. Analysis of growth kinetics by division tracking. Immunol. Cell Biol. 77, 523.

Overton, W.R., 1988. Modified histogram subtraction technique for analysis of flow cytometry data. Cytometry 9, 619. 
Parish, C.R., 1999. Fluorescent dyes for lymphocyte migration and proliferation studies. Immunol. Cell Biol. 77, 499.

Parish, C.R., Glidden, M.H., Quah, B.J., Warren, H.S., 2009. Use of the intracellular fluorescent dye CFSE to monitor lymphocyte migration and proliferation. Curr. Protoc. Immunol. 84, 4.9.1-4.9.13.

Pircher, H., Burki, K., Lang, R., Hengartner, H., Zinkernagel, R.M., 1989. Tolerance induction in double specific T-cell receptor transgenic mice varies with antigen. Nature 342, 559.

Quah, B.J., Parish, C.R., 2010. The use of carboxyfluorescein diacetate succinimidyl ester (CFSE) to monitor lymphocyte proliferation. J. Vis. Exp. (44), e2259. DOI:10.3791/2259.

Quah, B., Ni, K., O'Neill, H.C., 2004. In vitro hematopoiesis produces a distinct class of immature dendritic cells from spleen progenitors with limited T cell stimulation capacity. Int. Immunol. 16, 567.
Quah, B.J., Warren, H.S., Parish, C.R., 2007. Monitoring lymphocyte proliferation in vitro and in vivo with the intracellular fluorescent dye carboxyfluorescein diacetate succinimidyl ester. Nat. Protoc. 2, 2049.

Quah, B.J., Barlow, V.P., McPhun, V., Matthaei, K.I., Hulett, M.D., Parish, C.R., 2008. Bystander B cells rapidly acquire antigen receptors from activated B cells by membrane transfer. Proc. Natl. Acad. Sci. U. S. A. 105, 4259

Stambas, J., Doherty, P.C., Turner, S.J., 2007. An in vivo cytotoxicity threshold for influenza A virus-specific effector and memory CD8(+) T cells. J. Immunol. 178, 1285.

Weston, S.A., Parish, C.R., 1990. New fluorescent dyes for lymphocyte migration studies. Analysis by flow cytometry and fluorescence microscopy. J. Immunol. Methods 133, 87. 